\title{
CRÓNICA CONSTITUCIONAL DEL AÑO 2001
}

MIGUEL ÁNGEL PRESNO LINERA

Profesor Titular de Derecho Constitucional

Universidad de Oviedo 



\title{
CRÓNICA CONSTITUCIONAL DEL AÑO 2001
}

\author{
POR \\ MIGUEL ÁNGEL PRESNO LINERA \\ Profesor Titular de Derecho Constitucional \\ Universidad de Oviedo
}

Bajo esta rúbrica, y comenzando con el año 2001*, se inicia una sección en la que no se pretende más que realizar una síntesis expositiva de los principales acontecimientos políticos y jurídicos desarrollados en España a lo largo de cada año y que tengan una incidencia significativa en el ámbito constitucional. Se obviarán, salvo que se trate de sucesos de especial relevancia, aquellos hechos o datos que ya son objeto de tratamiento específico o de comentario en otras secciones de esta Revista o en publicaciones afines, cuya reiteración aquí sería superflua.

Hemos optado por realizar una presentación de carácter temático y no exclusivamente cronológica, por entender que así se puede ofrecer una panóramica más completa de los sucesos acaecidos, muchos de los cuales no se han concretado en un momento temporal único, sino que han tenido un desarrollo progresivo a lo largo de los doce meses examinados $o$, incluso, se extienden a años sucesivos. Como resulta obvio, dentro de cada sección o apartado sí se produce una ordenación temporal.

Los grandes bloques temáticos se refieren a la Unión Europea y las relaciones internacionales, los partidos políticos y los procesos

* La presente crónica se publica en el segundo número de los correspondientes al año 2002. En lo sucesivo, aparecerá en el número primero de cada año. 
electorales, a los órganos constitucionales, a la organización territorial del Estado y, por último, a los derechos, deberes y libertades de los ciudadanos.

Es necesario puntualizar de antemano que no se pretende llevar a cabo una exposición agotadora de todos los datos susceptibles de ser incluidos en las coordenadas mencionadas, sino seleccionar los más relevantes, lo que de manera inevitable conlleva el riesgo de que no estén todos los que son.

\section{UNIÓN EUROPEA Y RELACIONES INTERNACIONALES}

En estas materias hay que aludir, en primer término, a la creación, por Real Decreto 779/2001, de 5 de julio (BOE n. ${ }^{\circ} 167$, de 13 de julio), del Consejo para el Debate sobre el Futuro de la Unión Europea, dirigido a promover y estimular la participación de los ciudadanos y de las instituciones en el debate sobre el futuro de Europa. A través de la Red Derecons, y gracias a la gestión desarrollada por el Área de Derecho Constitucional de la Universidad de Oviedo, se puede participar en dicho debate y conocer los resultados del mismo; para más información: http://www.futuroeuropa.es/

Por la Ley Orgánica 3/2001, de 6 de noviembre (BOE $n .^{\circ} 267$, de 7 de noviembre), se autoriza la ratificación por España del Tratado de Niza, por el que se modifican el Tratado de la Unión Europea, los Tratados Constitutivos de las Comunidades Europeas y determinados actos conexos. El nuevo Tratado introduce importantes modificaciones que afectan a la composición y funcionamiento de las instituciones y órganos de la Unión, el sistema jurisdiccional, las mayorias cualificadas y cooperaciones reforzadas, los derechos fundamentales, seguridad y defensa, cooperación judicial penal y Tratado CECA. A partir del 1 de enero de 2005, entrará en vigor una reponderación de los votos atribuidos a cada Estado miembro en el Consejo, que tiene más en cuenta el factor demográfico, y también nuevas reglas para determinar la mayoría cualificada. En el Protocolo sobre la ampliación de la Unión Europea y declaraciones anejas se establecen las reglas y principios para fijar en los sucesivos tratados de adhesión los votos atribuidos a los nuevos Estados miembros y el umbral de la mayoría cualificada.

El Tratado establece una nueva composición de la Comisión que entrará en vigor a partir del año 2005 , modifica el modo de designa- 
ción de su Presidente y miembros, y refuerza considerablemente las competencias del Presidente. El sistema jurisdiccional de la Unión es objeto de una profunda reforma, de modo que el Tribunal de Justicia será el competente para conocer con carácter general los recursos prejudiciales y el garante de la unidad de la interpretación y aplicación del Derecho comunitario, mientras que el Tribunal de Primera Instancia será juez de Derecho común en materia de recursos directos. Se crean asimismo salas jurisdiccionales que conocerán de contenciosos muy especializados. Con vistas a la ampliación, el nuevo Tratado introduce también modificaciones relativas a la composición y organización del Tribunal de Cuentas, Comité Económico y Social, y Comité de las Regiones. En la Declaración relativa a la ampliación de la UE se determina, asimismo, el número de escaños que serán atribuidos en estos dos comités'a los nuevos Estados miembros en los futuros tratados de adhesión. Veintisiete disposiciones pasan íntegra o parcialmente de la unanimidad a la mayoría cualificada, y varias de éstas se regirán en adelante por el procedimiento de codecisión. Se facilita el recurso a las cooperaciones reforzadas en el ámbito del primer y del tercer pilar, y se abre la posibilidad de instaurarlas también en el campo de la política exterior y de seguridad común. El Tratado de la Unión Europea ha sido modificado a fin de reflejar en él los progresos realizados en el ámbito de la seguridad y defensa, para mencionar y describir las funciones de Eurojust, y establecer un mecanismo de alerta rápida para hacer frente a posibles riesgos de violaciones de los principios democráticos o de los derechos humanos por parte de un Estado miembro. Finalmente, un Protocolo establece las medidas necesarias para prever las consecuencias derivadas de la expiración del Tratado de la Comunidad Económica del Carbón y del Acero el 23 de julio de 2002.

En lo que tiene que ver con las relaciones internacionales de nuestro país, hay que referirse a algunos de los acuerdos y convenios a los que se ha incorporado España: en el BOE de 1 de marzo (.$^{\circ} 52$ ) se publicó el Instrumento de Ratificación del Protocolo Adicional al Convenio para la protección de los derechos humanos y la dignidad del ser humano con respecto a las aplicaciones de la biología y la medicina, por el que se prohíbe la clonación de seres humanos, hecho en París el 12 de enero de 1998. En el BOE de 12 de junio $\left(n .^{\circ} 140\right)$ se publicó el Instrumento de Ratificación del Convenio Internacional para la represión de los atentados terroristas cometidos con bombas, hecho en Nueva York el 15 de diciembre de 1997.

El Instrumento de Ratificación del Protocolo Facultativo de la Convención sobre la eliminación de todas las formas de discriminación 
contra la mujer, hecho en Nueva York el 6 de octubre de 1999, fue publicado en el BOE de 9 de agosto $\left(n .^{\circ} 190\right)$.

El 15 de septiembre se publicó (BOE $n .^{\circ} 222$ ) el Instrumento de Ratificación por España de la Carta Europea de las Lenguas Regionales o Minoritarias, hecha en Estrasburgo el 5 de noviembre de 1992. España declara que se entienden por tales las lenguas reconocidas como oficiales en los Estatutos de Autonomía de las Comunidades Autónomas del País Vasco, Cataluña, Illes Balears, Galicia, Comunidad Valenciana y Navarra; asimismo, también las que los Estatutos de Autonomía protegen y amparan en los territorios donde tradicionalmente se hablan.

El 29 de diciembre se publicó (BOE $n .^{\circ} 312$ ) el Instrumento de ratificación por España del Convenio Europeo sobre Indemnización a las Víctimas de Delitos Violentos, hecho en Estrasburgo el 24 de noviembre de 1983.

A través de una Resolución de 14 de diciembre, el Tribunal Supremo rechazó anular una condena en ejecución del Dictamen del Comité de Derechos Humanos de la ONU, que consideró vulnerado por España el derecho a la doble instancia penal establecido en el Pacto Internacional de Derechos Civiles y Políticos. De acuerdo con el criterio del Tribunal Supremo, el recurso de casación satisface la exigencia impuesta en el Pacto.

\section{PARTIDOS POLITIICOS Y PROCESOS ELECTORALES}

En lo que a los partidos políticos hace referencia, cabe diferenciar lo relativo a su organización y funcionamiento internos de lo que tiene que ver con el control externo.

Por lo que respecta a la primera cuestión, hay que destacar que el Partido Socialista Obrero Español (PSOE) celebró su Conferencia Política los días 20 a 22 de julio y en ella se acordó la implantación de un sistema de listas abiertas para la elección de los delegados a todos los congresos internos, así como la limitación temporal de tres mandatos seguidos en el mismo puesto institucional o del partido. En esta formación se integró el partido Nueva Izquierda después del acuerdo de autodisolución adoptado el $\mathbf{3 1}$ de marzo.

Convergència Democràtica de Catalunya y Unió Democràtica de Catalunya se convirtieron el 1 de abril en una federación de partidos, con el compromiso de celebrar una asamblea en 2004 para decidir a 
propósito de su fusión. El "número uno" de la federación será Artur Mas y el secretario Durán Lleida.

Respecto a los acuerdos entre las diferentes formaciones políticas, merece una atención especial el "Pacto de Estado para la Reforma de la Justicia», firmado el $\mathbf{3 1}$ de mayo entre el Partido Popular y el Partido Socialista, con el objetivo de que «la Justicia actúe con rapidez, eficacia y, calidad, con métodos más modernos y procedimientos menos complicados; que cumpla satisfactoriamente su función constitucional de garantizar en tiempo razonable los derechos de los ciudadanos y de proporcionar seguridad jurídica, al actuar con pautas de comportamiento y decisión previsibles, que actúe como poder independiente, unitario e integrado, con una estructura vertebrada, regida por una coherencia institucional que le permita desarrollar más eficazmente sus funciones constitucionales».

Entre las medidas concretas a desarrollar, los firmantes se comprometen a afrontar «las reformas necesarias para lograr un funcionamiento más ágil y eficaz del Tribunal Supremo y que potencien su función como órgano jurisdiccional superior y garante de la unidad de doctrina en todos los órdenes jurisdiccionales. A tal fin, también se reformará el recurso de casación, aproximando su regulación en las diferentes leyes reguladoras del proceso y atendiendo a la noción del «interés casacional». Se modificará el recurso de revisión, de manera que el Tribunal Supremo conozca exclusivamente de los "recursos de revisión contra sentencias firmes de la Audiencia Nacional y del propio Tribunal Supremo". Además, "se introducirán en la Ley Orgánica del Tribunal Constitucional mejoras que permitan potenciar su función con más eficacia y agilidad su función como interprete supremo de la Constitución, así como de garante de los derechos y libertades fundamentales consagrados en la misma. Ligada con esta reforma del recurso de amparo, se afrontarán mejoras sobre el desarrollo del artículo 53.2 de la Constitución y, más en general, sobre la protección de los derechos fundamentales por los Tribunales ordinarios". Por lo que se refiere a los Tribunales Superiores de Justicia, "la redefinición de sus competencias atenderá a criterios de adaptación de la Justicia al Estado de las Autonomías; estos Tribunales desarrollarán una función casacional en todas las ramas del Derecho Autonómico; se les atribuirá la segunda instancia penal, en los términos que resulten de la nueva Ley de Enjuiciamiento Criminal, y se aumentará la cuantía para el recurso de suplicación en el orden social; asimismo se trasladará a los Tribunales Superiores de Justicia la competencia para conocer de la petición de ejecución de sentencias extranjeras". Respecto al "Mapa Judicial", se aprobará uno nuevo, que "se establecerá con criterios que 
permitan ajustar la organización a la carga de trabajo existente en cada parte del territorio nacional. Se potenciará la Justicia de Proximidad, de manera que haya Juzgados más cercanos al ciudadano; se contemplará la creación de un nuevo órgano unipersonal: el Juzgado de lo Civil, que permitirá aligerar de su actual carga de trabajo a los Juzgados de Primera Instancia, será pieza esencial para acercar la Justicia al ciudadano; se avanzará en el diseño de juzgados especializados en materia mercantil". Entre otras medidas, "se elaborará un nuevo Estatuto de Jueces y Magistrados, así como que un nuevo régimen estatutario propio de la Carrera Fiscal».

Por lo que respecta a las actuaciones externas a los partidos pero referidas a ellos, hay que destacar la publicación de los Informes del Tribunal de Cuentas relativos a la fiscalización contable de los partidos políticos correspondientes a los años 1995, 1996 y 1997 (BOE de 26 de febrero, Suplemento del $n .^{\circ} 49$ ).

En los citados Informes se reiteran una vez más las críticas puestas de manifiesto con anterioridad (los estados financieros no incluyen la actividad completa de las organizaciones políticas, se computan recursos carentes de amparo legal, las entidades bancarias no prestan la colaboración suficiente...), lo que fleva al Tribunal de Cuentas a recomendar, entre otras cosas, que se regule "el marco normativo básico de la actividad de los partidos políticos" y se determine "de forma explícita el órgano y procedimiento a seguir en el régimen sancionador y en la exigencia de las responsabilidades previstas en la Ley Orgánica 3/1987, sobre finaciación de los partidos políticos».

A propósito de los procesos electorales internos, hemos de referirnos a las elecciones a los Parlamentos Vasco y de Galicia. En las primeras, celebradas el 13 de mayo con una participación del $79,9 \%$ del electorado, se dieron los siguientes resultados: la coalición Partido Nacionalista Vasco-Eusko Alkartasuna obtuvo 599.746 votos $(42,7 \%)$ y 33 escaños (6 escaños más que en las elecciones de 1998); el Partido Popular, 323.918 (23\%) y 19 escaños (1 más); el Partido Socialista de Euskadi, $250.919(17,8 \%)$ y 13 escanos (1 menos); Euskal Herritarrok, $142.784(10,1 \%)$ y 7 escaños ( 7 menos), e Izquierda Unida, 78.448 (5.5\%) y 3 escaños (1 más).

En las elecciones al Parlamento de Galicia, celebradas el 21 de octubre con una participación del $60,20 \%$ del electorado, se dieron los siguientes resultados: el Partido Popular obtuvo 791.885 votos $(51,60 \%)$ y 41 escaños (1 menos que en las elecciones de 1997); el Bloque Nacionalista Galego, $346.423(22,60 \%)$ y 17 escaños (1 menos), y el Partido Socialista de Galicia, $334.819(21,8 \%$ ) y 17 escaños (2 más). 


\section{ÓRGANOS CONSTITUCIONALES}

En lo relativo a las Cortes Generales, y por lo que respecta a su organización interna, debe mencionarse que el $\mathbf{3 0}$ de junio se publica en el BOE ( $^{\circ}$ 156) la Reforma del Reglamento del Congreso de los Diputados por la que se introduce un apartado 2 en el artículo $205 \mathrm{del}$ Reglamento de la Cámara para recoger el procedimiento de designación de los seis miembros del Consejo General del Poder Judicial que corresponde elegir a esta Cámara:

"La propuesta para el nombramiento de los 6 Vocales del Consejo General del Poder Judicial que la Cámara debe realizar entre Jueces y Magistrados de todas las categorías judiciales se ajustará a las siguientes reglas: a) La presentación de candidatos, hasta un máximo de 36, corresponderá a los Jueces y Magistrados, en los términos establecidos en la Ley Orgánica del Poder Judicial. b) Los candidatos presentados conforme a lo dispuesto en la letra a) serán sometidos directamente a la votación del Pleno, una vez comprobado por la Mesa de la Cámara que cumplen los requisitos constitucional y legalmente establecidos, sin que proceda la comparecencia previa de los mismos. c) Los Grupos parlamentarios podrán intervenir para explicar su posición por un tiempo máximo de 5 minutos. d) Las votaciones se ajustarán a lo dispuesto en los apartados 4 a 6 del artículo anterior, pudiendo cada Diputado escribir en la papeleta hasta 6 nombres".

En el mismo sentido, el nuevo apartado 6 del artículo 184 del Reglamento del Senado (BOE n. ${ }^{\circ} 155$, de 29 de junio) dispone que

"la propuesta para el nombramiento de los 6 Vocales del Consejo General del Poder Judicial que la Cámara debe realizar entre Jueces y Magistrados de todas las categorías judiciales se ajustará a las siguientes reglas: a) La presentación de candidatos, hasta un máximo de 36, corresponderá a los Jueces y Magistrados, en los términos establecidos en la Ley Orgánica del Poder Judicial. b) Los candidatos presentados conforme a lo dispuesto en la letra a), con excepción de los elegidos previamente por el Congreso de los Diputados, serán sometidos directamente a la votación del Pleno, una vez comprobado por la Mesa de la Cámara que cumplen los requisitos constitucional y legalmente establecidos, y sin que proceda la comparecencia prevista en el artículo siguiente. c) La deliberación y las votaciones se ajustarán a lo dispuesto en el artículo 186 de este Reglamento, salvo en lo dispuesto en su apartado 1 sobre el informe de presentación". 
Luego de la aprobación de las anteriores normas, las Cámaras procedieron a la designación de los Vocales del Consejo General del Poder Judicial, cuyos nombramientos, mediante los Reales Decretos 1222 y 1223 , se publicaron en el BOE de 7 de noviembre $\left(n .^{\circ} 267\right)^{1}$. En este mismo Boletín se incluyen los Acuerdos de las Cámaras por las que se designan Consejeros de Cuentas ${ }^{2}$.

En el Congreso de los Diputados también se procedió a la renovación de cuatro Magistrados del Tribunal Constitucional, cargos para los que resultaron elegidos D. Javier Delgado Barrio, D. Roberto García Calvo, D. Eugenio Gay Montalvo y Doña Elisa Pérez Vera (BOE de 7 de noviembre, $n .^{\circ} 267$ ), que sustituyen a D. Pedro Cruz Villalón, $D$. Carles Viver Pi-Sunyer, D. Julio Gónzalez Campos y D. Rafael Mendizábal Allende.

En el Boletín Oficial de las Cortes Generales (Senado) de 24 de mayo (.$^{\circ}$ 202) se publicó la Norma Supletoria de la Presidencia del Senado sobre desarrollo del artículo 163 del Reglamento del Senado, que regula las preguntas urgentes con respuesta oral en Pleno ${ }^{3}$.

1 Resultaron elegidos en el Congreso, D. Agustín Azparren, Dña. Montserrat Comas, D. Fernando Fernández, D. Adolfo Prego, D. José Luis Requero, D. Fernando Salinas, D. José Antonio Alonso, D. Faustino Gutiérrez-Alviz, D. Josep A. López y D. Félix Pantoja; en el Senado, D. Juan Carlos Campo, D. Juan Pablo González, D. Enrique López, D. Javier Martínez, D. José Merino, D. Enrique Míguez, D. Luis Aguiar, Dña. M.a Angeles García, D. Javier Laorden y D. Carlos Ríos.

2 A D. Ramón Álvarez, D. Luis Martínez, D. Manuel Núñez, Dna. Ana Pérez, D. Juan Velarde y D. Ciriaco de Vicente en el Congreso; a D. Lluis Armet, D. Rafael Corona, D. Felipe García, D. Javier Medina, D. Ubaldo Nieto y D. Antonio de la Rosa en el Senado.

3 Los Senadores presentarán estas preguntas en el Registro General de la Cámara entre el lunes y las doce horas del jueves de la semana anterior a aquélla en que se vaya a celebrar la sesión plenaria en que deban ser tramitadas. El escrito de presentación de estas preguntas contendrá una sola interrogante, sin necesidad de que contengan un preámbulo, exposición de motivos o introducción similar. La calificación de las preguntas corresponderá a la Mesa del Senado o a los miembros de la misma en quienes delegue.

La Mesa de la Cámara, oída la Junta de Portavoces, establecerá el número máximo de preguntas urgentes con respuesta oral en Pleno que puedeh incluirse en el orden del día de una șesión plenaria. En el supuesto de que se presenten más preguntas que las establecidas como máximo para una misma sesión plenaria, los criterios para determinar la prioridad de su inclusión en el orden del día serán los siguientes: a) Se incluirá en primer lugar una pregunta por cada uno de los Grupos Parlamentarios a los que pertenezcan los Senadores preguntantes. b) Para cubrir el resto hasta el número máximo fijado para una sesión plenaria se incluirán las del Senador perteneciente al Grupo Parlamentario que, durante el período 
En relación con la actividad política y de control del Congreso de los Diputados, se celebró el Debate sobre el Estado de la Nación (26 y 27 de junio), a resultas del cual se aprobaron 51 resoluciones de las 148 propuestas por los diferentes Grupos.

de sesiones, haya efectuado un menor consumo del cupo según el índice de proporcionalidad establecido por la Mesa del Senado, oída la Junta de Portavoces. Este indice de proporcionalidad se revisará al inicio de cada período de sesiones. Si el consumo de cupo entre dos o más Grupos Parlamentarios fuese el mismo, tendrá preferencia el Grupo con mayor número de Senadores miembros, y, en caso de igualdad, el que corresponda según el criterio de prelación que la Mesa establezca, oída la Junta de Portavoces. Dentro de cada Grupo Parlamentario, el Portavoz decidirá el orden de prioridad para la inclusión de las preguntas de sus miembros, entendiéndose que se ha optado por el criterio del orden de presentación si no se indicara otra cosa. No obstante, dentro del Grupo Mixto, el orden de presentación determinará la prelación de las preguntas. Las preguntas no incluidas en el orden del día del Pleno conforme al sistema establecido en esta Norma decaerán. Deberán presentarse de nuevo por quienes pretendan su formulación en una próxima sesión plenaria. El aplazamiento de una pregunta por el Gobierno determinará su inclusión en el orden del día de una sesión plenaria posterior, en los términos del artículo 163.3 del Reglamento del Senado. El Senador preguntante podrá ser sustituido por otro de su mismo Grupo Parlamentario, previa comunicación a la Presidencia por el Portavoz. Los Grupos Parlamentarios podrán sustituir cualquiera de las preguntas presentadas por los Senadores miembros del mismo e incluidas en el proyecto de orden del día del Pleno, conforme a lo dispuesto en las reglas precedentes por otras relativas a los acuerdos adoptados por el Consejo de Ministros, ajustándose al siguiente procedimiento: a) La solicitud de sustitución deberá presentarse en el Registro antes de las veinte horas del viernes en que el Consejo de Ministros haya tenido lugar. b) La solicitud especificará la pregunta sustituida, el texto de la nueva pregunta y el Senador miembro del Grupo que la formulará. c) La nueva pregunta deberá referirse a alguno de los acuerdos adoptados por el Consejo de Ministros. Comprobado el cumplimiento de los requisitos establecidos en el número anterior, la Mesa, o los miembros de la misma en quienes ésta delegue, acordarán la sustitución y lo pondrán inmediatamente en conocimiento del Gobierno. Los Grupos Parlamentarios podrán solicitar la sustitución de cualquiera de las preguntas presentadas por los Senadores miembros del mismo e incluidas en el proyecto de orden del día del Pleno conforme a lo dispuesto en las reglas precedentes por otras de especial actualidad o urgencia, ajustándose al siguiente procedimiento: a) La solicitud de sustitución deberá presentarse en el Registro antes de las doce horas del lunes de cada semana en que haya sesión plenaria en la que se vayan a tramitar preguntas. b) La solicitud especificará la pregunta sustituida, el texto de la nueva pregunta y el Senador miembro del Grupo que la formulará. c) La nueva pregunta sólo podrá referirse a hechos o circunstancias de especial actualidad o urgencia que no hayan podido ser objeto de pregunta en los plazos ordinarios. d) La petición de sustitución será remitida inmediatamente al Gobierno, para su conocimiento. Comprobado el cumplimiento de los requisitos establecidos para la sustitución, la Mesa de la Cámara ordenará la inclusión de la nueva pregunta en el orden del día del Pleno, previa audiencia de la Junta de Portavoces y con la conformidad del Gobierno. 
Asimismo, en la Cámara Baja se constituyó la Comisión de Investigación sobre "Gescartera", cuyo funcionamiento se desarrolló entre el 7 de septiembre y el 15 de noviembre. A resultas de este caso se produjeron las dimisiones de D. Enrique Giménez-Reyna como secretario de Estado de Hacienda, así como la de Dña. Pilar Valiente, Presidenta de la Comisión Nacional del Mercado de Valores.

Por lo que respecta a la actividad legislativa de las Cortes Generales, se aprobaron a lo largo de 2001 siete Leyes Orgánicas: Ley Orgánica $1 / 2001$, de 26 de marzo, por la que se modifica la Ley Orgänica 13/1982, de 10 de agosto, de reintegración y amejoramiento del Régimen Foral de Navarra (BOE n. 75 , de 28 de marzo); Ley Orgánica 2/2001, de 28 de junio, sobre composición del Consejo General del Poder Judicial, por la que se modifica la Ley Orgánica 6/1985, de 1 de julio, del Poder Judicial (BOE n. ${ }^{\circ} 155$, de 29 de junio); Ley Orgánica $3 / 2001$, de 6 de noviembre, por la que se autoriza la ratificación por España del Tratado de Niza por el que se modifican el Tratado de la Unión Europea, los Tratados constitutivos de las Comunidades Europeas y determinados actos conexos, firmado en Niza el día 26 de febrero de 2001 (BOE n. ${ }^{\circ} 267$, de 7 de noviembre); Ley Orgánica 4/2001, de 12 de noviembre, reguladora del Derecho de Petición (BOE n. ${ }^{\circ} 272$, de 13 de noviembre); Ley Orgánica 5/2001, de 13 de diciembre, complementaria a la Ley General de Estabilidad Presupuestaria (BOE n. ${ }^{\circ} 299$, de 14 de diciembre); Ley Orgánica 6/2001, dê 21 de diciembre, de Universidades (BOE n. ${ }^{\circ} 307$, de 24 de diciembre), y Ley Orgánica 7/2001, de 27 de diciembre, de modificación de la Ley Orgánica 8/1980, de 22 de septiembre, de Financiación de las Comunidades Autónomas (BOE n. ${ }^{\circ} 313$, de 31 de diciembre).

Además, se aprobaron veintiséis leyes ordinarias, entre las que cabe destacar las siguientes: Ley 3/2001, de 26 de marzo, de pesca marítima del Estado (BOE n. ${ }^{\circ} 75$, de 28 de marzo; corrección de erratas: BOE n. ${ }^{\circ} 174$, de 21 de julio); Ley $6 / 2001$ de 8 de mayo, de modificación del Real Decreto Legislativo 1302/1986, de 28 de junio, de evaluación de impacto ambiental (BOE n: 111, de 9 de mayo); Ley 7/2001, de 14 de mayo, de modificación de la Ley del Patrimonio de Estado, Texto Articulado aprobado por Decreto 1022/1964, de 15 de abril (BOE n. ${ }^{\circ} 116$, de 15 de mayo); Ley 10/2001, de 5 de julio, del Plan Hidrológico Nacional (BOE n. ${ }^{\circ} 161$, de 6 de julio de 2001; corrección de errores: $B O E n .^{\circ} 184$, de 2 de agosto); Ley 11/2001, de 5 de julio, por la que se crea la Agencia Española de Seguridad Alimentaria (BOE n. ${ }^{\circ} 161$, de 6 de julio); Ley 12/2001, de 9 de julio, de medidas urgentes de reforma del mercado de trabajo para el incremento del empleo y su calidad (BOE n. ${ }^{\circ} 164$, de 10 de julio); Ley 15/2001, de 9 de julio, de fomen- 
to y promoción de la cinematografía y el sector audiovisual (BOE $n$. $^{\circ}$ 164, de 10 de julio); Ley 17/2001, de 7 de diciembre, de Marcas (BOE n. ${ }^{\circ} 294$, de 8 de diciembre); Ley 18/2001, de 12 de diciembre, General de Estabilidad Presupuestaria (BOE n. ${ }^{\circ} 298$, de 13 de diciembre; corrección de errores: $B O E n .^{\circ} 40$, de 15 de febrero de 2002); Ley 19/2001, de 19 de diciembre, de reforma del Texto Articulado de la Ley sobre Tráfico, Circulación de Vehículos a Motor y Seguridad Vial, aprobado por Real Decreto Legislativo 339/1990, de 2 de marzo (BOE n. ${ }^{\circ} 308$, de 20 de diciembre); Ley 22/2001, de 27 de diciembre, reguladora de los Fondos de Compensación Interterritorial (BOE n. ${ }^{\circ} 313$, de 31 de diciembre); Ley 23/2001, de 27 de diciembre, de Presupuestos Generales del Estado para el año 2002 (BOE n. ${ }^{\circ} 313$, de 31 de diciembre); Ley 24/2001, de 27 de diciembre, de Medidas Fiscales, Administrativas y del Orden Social (BOE n. ${ }^{\circ} 313$, de 31 de diciembre), y Ley 25/2001, de 27 de diciembre, por la que se prorroga la vigencia del Concierto Económico con la Comunidad Autónoma del País Vasco aprobado por Ley 12/1981, de 13 de mayo (BOE n. ${ }^{\circ} 313$, de 31 de diciembre) ${ }^{4}$.

4 Las otras Leyes aprobadas son las siguientes: Ley 1/2001, de 13 de marzo, por la que se autoriza la participación del Reino de España en la octava reposición de recursos del Fondo Africano de Desarrollo (BOE n. ${ }^{\circ} 64$, de 15 de marzo); Ley $2 / 2001$, de 26 de marzo, por la que se crea el Consejo General de Colegios de Profesionales Dentales (BOE n..$^{\circ}$, de 28 de marzo); Ley 4/2001, de 24 de abril, sobre concesión de un crédito extraordinario, por importe de 15.560 .625 .000 pesetas, para compensar el déficit de la Compañía Transmediterránea, Sociedad Anónima, correspondiente al ejercicio de 1997, por la explotación de los servicios de comunicaciones marítimas de interés nacional y los ajustes por la finalización del contrato regulador de los referidos servicios (BOE $n .^{\circ} 99$, de 25 de abril); Ley 5/2001, de 8 de mayo, sobre concesión de un suplemento de crédito, por importe de 4.866.309.925 pesetas, para el pago de retribuciones establecidas en diversas sentencias firmes, falladas a favor de las personas encargadas de la enseñanza de la religión católica en los colegios públicos (BOE $n .^{\circ} 111$, de 9 de mayo); Ley $8 / 2001$, de 4 de junio, sobre concesión de un crédito extraordinario, por un importe de 13.858.385.705 pesetas, para atender insuficiencias de crédito producidas en el ejercicio de 1999, por las subvenciones que se efectúan para la cobertura de las diferencias de cambio de las divisas obtenidas en préstamos concedidos a la sociedades concesionarias de autopistas de peaje (BOE $n .^{\circ} 134$, de 5 de junio); Ley $9 / 2001$, de 4 de junio, por la que se modifica la disposición transitoria sexta de la Ley 54/1997, de 27 de noviembre, del Sector eléctrico determinados artículos de la Ley 16/1989, de 17 de julio, de Defensa de la Competencia y determinados artículos de la Ley 4611988, de 17 de diciembre, sobre introducción del euro (BOE n. ${ }^{\circ}$ 134, de 5 de junio); Ley 13/2001, de 9 de julio, sobre concesión de un crédito extraordinario, por importe de 8.229.849.001 pesetas, para atender el pago de obligaciones derivadas de la compensación a las Universidades públicas por la reducción o exención en los precios publicos por servicios académicos a alumnos de familias numerosas correspondientes a los cursos 19961997 y 1997-1998 (BOE n. ${ }^{\circ}$ 
A propósito de la Jefafura del Estado ha de mencionarse el Real Decreto 1033/2001, de 21 de septiembre, por el que se modifican el Real Decreto 434/1988, de 6 de mayo, sobre la reestructuración de la Casa de Su Majestad el Rey, y el Real Decreto 725/1993, de 14 de mayo, por el que se modifica el Real Decreto 2157/1977, de 23 de julio, de creación del distintivo de la Casa de Su Majestad el Rey (BOE $n .^{\circ} 228$, de 22 de septiembre).

En el Gobierno de la Nación se produjo una remodelación luego del cese, a petición propia, del Ministro del Interior, D. Jaime Mayor Oreja (Real Decreto 204, de 27 de febrero), que fue sustituido por D. Mariano Rajoy Brey, nombrado por Real Decreto 207, de 27 de febrero. A su vez, por Decreto 108, de la misma fecha, fue nombrado Ministro de la Presidencia D. Juan José Lucas Jiménez. A través del Real Decreto 209 (BOE n. ${ }^{\circ}$ 51, de 28 de febrero) se modifica el Real Decreto $558 / 2000$ sobre las Vicepresidencias del Gobierno, estableciéndose que corresponden a la Vicepresidencia Primera las funciones que le encomiende el Presidente del Gobierno.

Por lo que a la actividad legislativa del Gobierno se refiere, el Ejecutivo aprobó un Decreto Legislativo, el Real Decreto Legislativo $1 / 2001$, de 20 de julio, por el que se aprueba el Texto Refundido de la Ley de Aguas (BOE n. ${ }^{\circ} 176$, de 24 de julio), y dieciséis Decretos-Ley, pudiendo destacarse los siguientes: Real Decreto-ley 2/2001, de 2 de febrero, por el que se modifica la disposición transitoria sexta de la Ley 54/1997, de 27 de noviembre, del Sector Eléctrico y determinados artículos de la Ley 16/1989, de 17 de julio, de Defensa de la Competencia (BOE $n .{ }^{\circ} 30$, de 3 de febrero; convalidación: 22 de febrero, BOE n. ${ }^{\circ}$ 51, de 28 de febrero); Real Decreto-ley 5/2001, de 2 de marzo, de medidas urgentes de reforma del Mercado de Trabajo para el incremento del empleo y la mejora de su calidad (BOE $n .^{\circ} 54$, de 3 de marzo de 2001; corrección de errores: $B O E$ n. ${ }^{\circ} 60$, de 10 de marzo; convalidación:

164, de 10 de julio); Ley 14/2001, de 9 de julio, sobre concesión de dos créditos extraordinarios por importe total de 4.246 .984 .705 pesetas (25.524.892 euros) para el pago de las cantidades derivadas de la sentencia del Tribunal Superior de Justicia de Andalucía, de 28 de diciembre de 1998, a los titulares de varias fincas expropiadas en el Parque Nacional de Doñana (BOE $n .^{\circ} 164$, de 10 de julio); Ley 16/2001, de 21 de noviembre, por la que se establece un proceso extraordinario de consolidación y provisión de plazas de personal estatutario, en las Instituciones Sanitarias de la Seguridad Social de los Servicios de Salud del Sistema Nacional de Salud (BOE n. ${ }^{\circ} 280$, de 22 de noviembre); Ley 26/2001, de 27 de diciembre, por la que se establece el sistema de infracciones y sanciones en materia de encefalopatias espongiformes transmisibles (BOE n. 7 , de 2 de enero de 2002). 
15 de marzo de 2001, BOE n. ${ }^{\circ} 72$, de 24 de marzo de 2001); Real Decreto-ley $11 / 2001$, de 22 de junio, por el que se modifica el artículo 29 de la Ley 25/1988, de 29 de julio, de Carreteras, y se establecen normas presupuestarias para atender los gastos derivados de actuaciones del Ministerio de Fomento en carreteras estatales (BOE $n .^{\circ} 150$, de 23 de junio; convalidación: 28 de junio, $B O E n .^{\circ} 156$, de 30 de junio); Real Decreto-ley 15/2001, de 2 de noviembre, por el que se adoptan medidas urgentes en materia de transporte aéreo (BOE $n .{ }^{\circ} 264$, de 3 de noviembre; convalidación: 22 de noviembre, BOE n. ${ }^{\circ} 286$, de 29 de noviembre); y el Real Decreto-ley 16/2001, de 27 de diciembre, de medidas para el establecimiento de un sistema de jubilación gradual y flexible (BOE n..$^{\circ} 313$, de 31 de diciembre; convalidación: 5 de febrero de 2002, BOE n. ${ }^{\circ} 38$, de 13 de febrero de 2002).

En lo que se refiere al Poder Judicial, tras la elección del nuevo Consejo General del Poder Judicial llevada a cabo por las Cortes Generales 5 , el Pleno eligió como Presidente al Magistrado de la Sala de lo Contencioso-Administrativo del Tribunal Supremo, D. Francisco José Hernando Santiago, publicándose su nombramiento en el Real Decreto 1224 , de 7 de noviembre, en el BOE n. ${ }^{\circ} 268$, de 8 de noviembre.

La elección por las Cámaras del Pleno del Consejo se desarrolló de acuerdo con los nuevos criterios introducidos por la Ley Orgánica $2 / 2001$, de 28 de junio, sobre composición del Consejo General del Poder Judicial, por la que se modifica la Ley Orgánica 6/1985, de 1 de julio, del Poder Judicial (BOE n. ${ }^{\circ} 155$, de 29 de junio) ${ }^{6}$. Sobre este par-

\section{Véase la nota 1.}

6 Según dispone el nuevo artículo 112 de la LOPJ, «Los 12 miembros que conforme a lo dispuesto en el artículo 122 de la Constitución han de integrar el Consejo entre Jueces y Magistrados de todas las categorías judiciales serán propuestos para su nombramiento por el Rey de acuerdo con el siguiente procedimiento: Podrán ser propuestos los Jueces y Magistrados de todas las categorías judiciales que se hallen en servicio activo y no sean miembros del Consejo saliente o presten servicios en los órganos técnicos del mismo. La propuesta será formulada al Rey por el Congreso de los Diputados y el Senado, correspondiendo a cada Cámara proponer seis Vocales, por mayoria de tres quintos de sus respectivos miembros, entre los presentados a las Cámaras por los Jueces y Magistrados conforme a lo previsto en el número siguiente.

Los candidatos serán presentados, hasta un máximo del triple de los doce puestos a proponer, por las asociaciones profesionales de Jueces y Magistrados o por un número de Jueces y Magistrados que represente, al menos, el $2 \%$ de todos los que se encuentren en servicio activo. La determinación del número máximo de candidatos que corresponde presentar a cada asociación y del número máximo de candidatos que pueden presentarse con las firmas de Jueces y 
ticular, ha de citarse la Instrucción de 29 de junio del Presidente del Consejo General del Poder Judicial por la que se determina el número de candidatos a presentar por las Asociaciones Profesionales de Jueces y Magistrados, y se concretan otros aspectos de formulación de candidaturas a Vocal del Consejo (BOE n. ${ }^{\circ} 156$, de 20 de junio).

En lo que respecta al ejercicio de las funciones jurisdiccionales, el 7 de enero de 2001 entró en vigor la Ley $1 / 2000$, de 7 de enero (BOE n. ${ }^{\circ} 7$, de 8 de enerol, de Enjuiciamiento Civil. Por Acuerdo Reglamentario 4/2001, de 6 de noviembre, del Pleno del Consejo General del Poder Judicial, se modifica el Reglamento $1 / 1995$, de 7 de junio, de la Carrera Judicial, en lo relativo a Magistrados suplentes y Jueces sustitutos (BOE n. ${ }^{\circ} 279$, de 21 de noviembre).

Por lo que al Tribunal Constitucional se refiere, por Acuerdo de 18 de enero de 2001 (BOE de 23 de enero, n. $^{\circ} 20$ ) del Pleno del Tribunal, se modifica el horario del Registro General del Tribunal Constitucional, disponiéndose que el Registro estará abierto todos los días hábiles, incluso durante el mes de agosto, desde las nueve treinta hasta las quince horas, en la sede del mismo.

Magistrados se ajustará a criterios estrictos de proporcionalidad, de acuerdo con las siguientes reglas:

Los 36 candidatos se distribuirán en proporción al número de afiliados de cada asociación y al número de no afiliados a asociación alguna, determinando este último el número máximo de candidatos que pueden ser presentados mediante firmas de otros Jueces y Magistrados no asociados; todo ello, de acuerdo con los datos obrantes en el Registro constituido en el Consejo General del Poder Judicial conforme a lo previsto en el artículo $\mathbf{4 0 1}$ de la presente Ley Orgánica y sin que ningún Juez o Magistrado pueda avalar con su firma más de un candidato.

En el caso de que el número de Jueces y Magistrados presentados con el aval de firmas suficientes supere el máximo al que se refiere la letra a), sólo.tendrán la consideración de candidatos los que, hasta dicho número máximo, vengan avalados por el mayor número de firmas. En el supuesto contrario de que el número de candidatos avalados mediante firmas no baste para cubrir el número total de 36, los restantes se proveerán por las asociaciones, en proporción al número de afiliados; a tal efecto y para evitar dilaciones, las asociaciones incluirán en su propuesta inicial, de forma diferenciada, una lista complementaria de candidatos.

Cada asociación determinará, de acuerdo con lo que dispongan sus Estatutos, el sistema de elección de los candidatos que le corresponda presentar.

Entre los 36 candidatos presentados, conforme a lo dispuesto en el número anterior, se elegirán en primer lugar 6 Vocales por el Pleno del Congreso de los Diputados, y una vez elegidos estos 6 Vocales, el Senado elegirá los otros 6 entre los 30 candidatos restantes. Todo ello sin perjuicio de lo dispuesto en el número 2 del artículo siguiente". 
Como ya se ha dicho, el Congreso de los Diputados procedió a la renovación de los cuatro Magistrados que le corresponde elegir; resultaron nombrados D. Javier Delgado Barrio, D. Roberto García Calvo, D. Eugenio Gay Montalvo y Doña Elisa Pérez Vera (BOE de 7 de noviembre, $n .^{\circ}$ 267), que sustituyen a D. Pedro Cruz Villalón, D. Carles Viver PiSunyer, D. Julio Gónzalez Campos y D. Rafael Mendizábal Allende.

Se procedió, asimismo, a la elección de nuevo Presidente y Vicepresidente, recayendo dichos cargos en D. Manuel Jiménez de Parga y D. Tomás Vives Antón (Reales Decretos 1244 y 1255, BOE n. ${ }^{\circ} 273$, de 14 de noviembre).

Por Acuerdo de 13 de noviembre del Pleno del Tribunal (BOE n. ${ }^{\circ}$ 274 , de 16 de noviembre), se dispone la composición de las Salas y Secciones. La Sala Primera estará integrada por D. Manuel Jiménez de Parga, Presidente, y D. Pablo García Manzano, D. Fernando Garrido Falla, Dña. María Emilia Casas Baamonde, D. Javier Delgado Barrio y D. Roberto García Calvo. La Sala Segunda, por D. Tomás Vives Antón, Presidente, y D. Pablo Cachón Villar, D. Vicente Conde Martín de Hijas, D. Guillermo Jiménez Sánchez, Dña. Elisa Pérez Vera y D. Eugenio Gay Montalvo.

Por lo que se refiere a la tarea del Tribunal como "legislador negativo", han de mencionarse las siguientes sentencias: la STC 9/2001, de 18 de enero de 2001, estima parcialmente el recurso de inconstitucionalidad promovido por el Presidente del Gobierno frente a la Ley del Parlamento de Galicia 6/1993, de 11 de mayo, de Pesca de Galicia, $y$, en consecuencia: a) declara que vulneran el orden constitucional de competencias y resultan, por consiguiente, nulos: - El primer párrafo de la letra a) del art. 6 , en lo que respecta a la elaboración de los "planes de pesca». - El cuarto párrafo de la letra d) del art. 6.El art. $6 \mathrm{n}$ ); b) declara que no vulnera el orden constitucional de competencias, interpretado en el sentido que se indica en el fundamento jurídico 11, el art. 30.

La STC 62/2001, de 1 de marzo de 2001, estima parcialmente el recurso de inconstitucionalidad promovido por el Presidente del Gobierno contra determinados preceptos de la Ley de las Cortes de Aragón 7/1993, de 4 de mayo, de Presupuestos de la Comunidad Autónoma para 1993, y en su virtud: declara inconstitucionales y nulos los arts.17.1, 19.2 y 22.1, el apartado 1 de la Disposición adicional duodécima y el inciso final de la Disposición transitoria primera (uincrementadas en el porcentaje que con carácter general se apruebe para el personal funcionario en la presente Ley"), todos ellos de la Ley autonómica impugnada. 
La STC 97/2001, de 5 de abril de 2001, declara: $10^{\circ}$ que los incisos "en cada capital de provincia" y «en su correspondiente ámbito territorial" del art. 9 y "están presididas por un miembro del Ministerio Fiscal» y "actuando uno de ellos como Secretario" del art. 10.1 de la Ley $1 / 1996$, de 10 de enero, de Asistencia Jurídica Gratuita, vulneran las competencias de la Generalidad de Cataluña y, por ello, no son de aplicación en el ámbito territorial de la Comunidad Autónoma de Cataluña; $2{ }^{\circ}$ declara que la referencia a los citados incisos de los arts. 9 y 10.1 contenida en la Disposición adicional primera, 1, vulnera las competencias de la Generalidad de Cataluña y, por ello, no es de aplicación en el ámbito territorial de la Comunidad Autónoma de Cataluña.

En la STC 115/2001, de 10 de mayo, que estima el recurso de amparo n. $.^{\circ} 1786 / 98$, la Sala plantea ante el Pleno del Tribunal cuestión de inconstitucionalidad respecto de los artículos 108, párrafo 2, de la Ley Orgánica 4/1987, de la Competencia y Organización de la Jurisdicción Militar, y 127, párrafo 1, de la Ley Orgánica 2/1989, de 13 de abril, Procesal Militar, por su contradicción con los arts. 14 y $24.1 \mathrm{CE}$, en relación con el inciso final del art.117.5 de la Constitución.

La STC 159/2001, de 5 de julio de 2001: $\left.1 .^{\circ}\right)$ Declara inconstitucional y, consiguientemente, nulo, el art. 15 del Decreto Legislativo de la Generalidad de Cataluña 1/1990, de 12 de julio, por el que se aprobó el texto refundido de los textos legales vigentes en Cataluña en materia urbanística. $\left.22^{\circ}\right)$ Declara inconstitucional y derogado por la Constitución el art. 218 del Real Decreto 1346/1976, de 9 de abril, por el cual se aprobó el texto refundido de la Ley sobre Régimen de Suelo y Ordenación Urbana. 3. ) Declara que el art. 47 del Decreto Legislativo de la Generalidad de Cataluña 1/1990, de 12 de julio, sólo es constitucional si se interpreta en el sentido de que la facultad de la Comunidad Autónoma de Cataluña de formular Planes de Conjunto únicamente puede llevarse a cabo «en defecto de acuerdo entre las Corporaciones Locales afectadas". $\left.4 .^{\circ}\right)$ Declara inconstitucional y derogado por la Constitución el art. 91.2 del Real Decreto 1346/1976, de 9 de abril, por el cual se aprobó el texto refundido de la Ley sobre Régimen de Suelo y Ordenación Urbana.

La STC 164/2001, de 11 de julio de 2001: 1. ${ }^{\circ}$ Declara que los arts. $9 ; 11 ; 14.2$ a) y b); $15 ; 17.1 ; 18.1,2$ y 3 de la Ley $6 / 1998$, de 13 de abril, sobre Régimen del Suelo y Valoraciones, en cuanto contienen las expresiones "planeamiento general", "planeamiento de desarrollo", "planes de ordenación territorial", "legislación sectorial», "planeamiento sectorial", "sistemas generales" o "dotaciones públicas de 
carácter local" son conformes con la Constitución siempre que se interpreten de conformidad con lo expresado, respectivamente, en los FFJJ $14,16,21,25,29$ y $30.2^{\circ}$ ) Declara que el art. 16.1 de la Ley sobre Régimen del Suelo y Valoraciones es inconstitucional y por tanto nulo. $3 .^{\circ}$ ) Declara que el art. 38 de la Ley sobre Régimen del Suelo y Valoraciones es inconstitucional y por tanto nulo. $\left.4 .^{\circ}\right)$ Declara la inconstitucionalidad de la Disposición final única de la Ley sobre Régimen del Suelo y Valoraciones, en sus referencias al art. 38 y al art. 16.1.

La STC 200/2001, de 4 de octubre de 2001, declara la inconstitucionalidad y nulidad del inciso «para que la adopción pueda surtir efectos pasivos es preciso que el adoptante haya sobrevivido dos años, al menos, desde la fecha de la adopción" del art. 41.2 del texto refundido de la Ley de Clases Pasivas del Estado, aprobado por Real Decreto Legislativo 670/1987, de 30 de abril.

La STC 206/2001, de 22 de octubre de 2001, estima parcialmente los recursos de inconstitucionalidad acumulados frente a la Ley 3/1993, de 22 de marzo, básica de las Cámaras Oficiales de Comercio, Industria y Navegación, y en su virtud: 1. ) Declara que el art. 16.2, en su inciso «La tercera parte restante estará afectada a la financiación de la función de colaboración con las Administraciones competentes en las tareas de formación a que se refiere el párrafo f) del apartado 1 y el párrafo d) del apartado 2 del artículo 2 de la presente Ley", así como la Disposición final segunda, apartado 2, no son aplicables en Cataluña ni en la Comunidad Valenciana. $2 .^{\circ}$ ) Declara que los arts. 3.3 y 18.2 h) sólo son aplicables en Cataluña y en la Comunidad Valenciana en los términos expuestos en los fundamentos jurídicos 9 y 11, respectivamente. $\left.3^{\circ}\right)$ Declara que el art. 3.4 es sólo aplicable en la Comunidad Valenciana en los términos expuestos en el fundamento juridico $\left.10.4 .^{\circ}\right)$ Declara que el art. 16.3 sólo es aplicable en Cataluña y en la Comunidad Valenciana en el sentido expresado en el fundamento jurídico $\left.21.5 .^{\circ}\right)$ Declara que los arts. 22.1, última frase, y 22.2, párrafo 2, sólo son aplicables en Cataluña y en la Comunidad Valenciana interpretados en el sentido expresado en el fundamento jurídico 12.6..$^{\circ}$ Declara que la Disposición final primera no es aplicable en Cataluña ni en la Comunidad Valenciana en la medida en que declara básicos los preceptos anteriormente declarados inaplicables.

La STC 234/2001, de 13 de diciembre de 2001, declara inconstitucional y nulo el apartado séptimo de la Disposición transitoria cuarta de la Ley 45/1985, de 23 de diciembre, de impuestos especiales. 
El Defensor del Pueblo presentó el 2 de octubre el Informe correspondiente a la gestión realizada durante el año 2000 (Boletín de las Cortes Generales, Serie A, n. ${ }^{\circ} 201$, de 8 de octubre).

En el Tribunal de Cuentas, además de la renovación de sus componentes, se procedió al nombramiento de su Presidente, resultando elegido para el cargo D. Ubaldo Nieto de Alba (BOE $n .^{\circ} 274$, de 15 de noviembre).

\section{ORGANIZACIÓN TERRITORIAL DEL ESTADO}

Por lo que se refiere al "Estado autonómico», se aprobó la Ley Orgánica 1/2001, de 26 de marzo, por la que se modifica la Ley Orgánica 13/1982, de 10 de agosto, de reintegración y amejoramiento del Régimen Foral de Navarra (BOE $n .^{\circ} 75$, de 28 de marzo).

Como ya se ha indicado, se celebraron las elecciones a los Parlamentos Vasco y de Galicia. En las primeras, celebradas el 13 de mayo con una participación del $79,9 \%$ del electorado, se dieron los siguientes resultados: la coalición Partido Nacionalista Vasco-Eusko Alkartasuna obtuvo 599.746 votos $(42,7 \%)$ y 33 escaños ( 6 escaños más que en las elecciones de 1998); el Partido Popular, $323.918(23 \%)$ y 19 escaños (1 más); el Partido Socialista de Euskadi, $250.919(17,8 \%)$ y 13 escanos ( 1 menos); Euskal Herritarrok, $142.784(10,1 \%)$ y 7 escaños (7 menos), e Izquierda Unid,a 78.448 (5,5\%) y 3 escaños (1 más). El 6 de julio el Partido Nacionalista Vasco y Eusko Alkartasuna firmaron un Acuerdo de gobierno y el 12 de julio fue investido como Lehendakari D. Juan José Ibarretxe Marcuartu. El 15 de septiembre se incorporó al Acuerdo de gobierno Izquierda Unida, lo que supuso una remodelación del Gobierno Vasco y la entrada en él de un Consejero de Izquierda Unida.

El 29 de enero el Parlamento de Galicia rechazó la moción de censura presentada por el Bloque Nacionalista Galego contra el Presidente de la Xunta, D. Manual Fraga. La moción, que incluía como candidato a Presidente a D. Xosé Manuel Beiras, contó con el voto favorable de los diputados del BNG; fue rechazada por los diputados del PP; los diputados del PSOE se abstuvieron.

En las elecciones al Parlamento de Galicia, celebradas el 21 de octubre con una participación del $60,20 \%$ del electorado, se dieron los siguientes resultados: el Partido Popular obtuvo 791.885 votos $(51,60 \%)$ y 41 escaños (1 menos que en las elecciones de 1997); el Bloque 
Nacionalista Galego, $346.423(22,60 \%)$ y 17 escaños ( 1 menos), y el Partido Socialista de Galicia, $334.819(21,8 \%$ ) y 17 escaños (2 más). El día 5 de diciembre fue investido como Presidente de la Xunta D. Manuel Fraga Iribarne.

El 19 de enero, Artur Mas tomó posesión como conseller en cap de la Generalitat de Cataluña.

El 6 de febrero prospera una moción de censura presentada contra el Alcalde-Presidente de Ceuta, D. Antonio Sampietro, del Grupo Independiente Liberal, y accede al cargo el Candidato alternativo D. Juan Jesús Vivas, del Partido Popular.

En Aragón, el 31 de octubre, Izquierda Unida firmó un Acuerdo de legislatura con el Partido Socialista y el Partido Aragonés Regionalista, aunque no entró a formar parte del Gobierno integrado por estas dos últimas formaciones.

El 27 de julio, el Gobierno y las 15 Comunidades Autónomas de régimen común acordaron, en el seno del Consejo de Política Fiscal y Financiera, un nuevo modelo de financiación autonómica que abandona el sistema quinquenal e introduce uno de vigencia indefinida. Además, se aprueba la cesión de la gestión del 33\% del IRPF a las Comunidades Autónomas; del $35 \%$ del IVA y, en diversos porcentajes, de los Impuestos Especiales. Otros acuerdos consistieron en la creación de una tasa sobre el consumo de gasolinas, destinada a financiar la sanidad, y la de un fondo de cohesión sanitaria para los ciudadanos desplazados de su Comunidad.

Sobre estas materias, en el BOE de 31 de diciembre, $n .{ }^{\circ} 313$, se publican las siguientes normas: la Ley Orgánica 7/2001, de 27 de diciembre, de modificación de la Ley Orgánica 8/1980, de 22 de septiembre, de Financiación de las Comunidades Autónomas; la Ley $21 / 2001$, de 27 de diciembre, por la que se regulan las medidas fiscales y administrativas del nuevo sistema de financiación de las Comunidades Autónomas de régimen común y Ciudades con Estatuto de Autonomía, y la Ley 22/2001, de 27 de diciembre, reguladora de los Fondos de Compensación Interterritorial.

Además, en ese mismo BOE se incluye la Ley 25/2001, de 27 de diciembre, por la que se prorroga la vigencia del Concierto Económico con la Comunidad Autónoma del País Vasco, aprobado por Ley 12/1981, de 13 de mayo.

El 26 de diciembre se firma el traspaso de las competencias en materia de sanidad a las Comunidades Autónomas que no la habian 
recibido; los Decretos en los que se formaliza el traspaso se publican en el BOE los días 28,29 y 31 de diciembre.

En el ámbito jurisprudencial, pueden mencionarse, entre otras, la STC 45/2001, de 15 de febrero de 2001, que estima parcialmente los conflictos 624/93 y 757/93 y, en consecuencia, declara que corresponde a las Comunidades Autónomas de Cantabria y Cataluña la competencia para adoptar la resolución final de los expedientes de asignación individual de las cantidades de referencia que no procedan de la reserva nacional y, por lo tanto, declarar inconstitucional y nulo el art. 1 del Real Decreto 1319/1992 en cuanto atribuye a la Dirección General correspondiente del Ministerio de Agricultura, Pesca y Alimentación la asignación de dichas cantidades; declara que corresponde al Estado la competencia para adoptar la resolución final en los expedientes de asignación de cantidades procedentes de la reserva nacional.

La STC 98/2001, de 5 de abril de 2001, declara que las competencias controvertidas contenidas en los arts. 4 , apartados 1 y $2 ; 6 ; 7$; $10 ; 11 ; 12$, salvo el primer párrafo del apartado $1 ; 13,14 ; 15$, salvo el inciso "y a las previstas en la legislación del Tribunal de Cuentas»; 16, y 17, de la Orden del Ministerio de Industria y Energía, de 7 de febrero de 1997, que aprueba las bases reguladoras de la concesión de subvenciones en el marco del Plan de ahorro y eficiencia energética (PAEE) para el período 1997-1999 y convoca las del ejercicio 1997, que corresponden a la Generalidad de Cataluña.

La STC 188/2001, de 20 de septiembre de 2001, $1 .^{\circ}$, estima parcialmente el conflicto positivo de competencia núm. 3386/94, declarando que vulneran las competencias de la Generalidad de Cataluña los arts. 15, segundo párrafo, $25,40,41,42.2$ y $3,43.2$ y $3,49.2,51,52$, 55 a 58 y el inciso "a la Dirección General de Formación Profesional Reglada y Promoción Educativa" del art. 62.1 de la Orden del Ministerio de Educación y Ciencia de 15 de junio de 1994 por la que se convocan becas y ayudas al estudio de carácter general para estudios universitarios y medios para el curso académico 1994-1995; $2 .^{\circ}$ estima parcialmente el conflicto positivo de competencia núm. 4710/97, declarando que vulneran las competencias de la Generalidad de Cataluña los arts. 15 , segundo párrafo, 25, 40,51,54,55.1, 56, 57 y el inciso «a la Dirección General de Formación Profesional y Promoción Educativa" del art. 62.1 de la Orden de 30 de junio de 1997 del Ministerio de Educación y Cultura, por la que se convocan becas y ayudas al estudio de carácter general, para estudios universitarios y medios para el curso 1997-1998. 


\section{DERECHOS, DEBERES Y LIBERTADES}

En esta cuestión se podrían clasificar los hechos más relevantes atendiendo a su impronta normativa y a su carácer jurisprudencial. En el primer ámbito habría que referirse, a grandes rasgos, a las numerosas normas aprobadas a lo largo del año 2001.

En materia de extranjeria e inmigración, se crea el Consejo Superior de Política de Inmigración (Real Decreto 344/2001, de 4 de abril; BOE $n .^{\circ} 83$, de 6 de abril) y se regula el Observatorio Permanente de la Inmigración (Real Decreto 345/2001, de 4 de abril; BOE n. ${ }^{\circ} 83$, de 6 de abril). A través del Real Decreto 864/2001, de 20 de julio (BOE $n$. $^{\circ}$ 174, de 21 de julio), se aprueba el Reglamento de ejecución de la Ley Orgánica 4/2000, de 11 de enero, sobre Derechos y Libertades de los Extranjeros en España y su integración social, reformada por la Ley Orgánica 8/2000, de 22 de diciembre. Por Real Decreto 865/2001, de 20 de julio, se aprueba el Reglamento de reconocimiento del Estatuto de apátrida (BOE $n .^{\circ} 174$, de 21 de julio; corrección de errores en BOE n. ${ }^{\circ}$ 276, de 17 de noviembre).

Sobre libertad personal y seguridad, cabe mencionar la Ley 19/2001, de 19 de diciembre, de reforma del Texto Articulado de la Ley sobre Tráfico, Circulación de Vehículos a Motor y Seguridad Vial, aprobado por Real Decreto Legislativo 339/1990, de 2 de marzo (BOE n. ${ }^{\circ}$ 308, de 20 de diciembre). Por Real Decreto 1123/2001, de 19 de octubre (BOE $n .^{\circ} 281$, de 23 de noviembre), se modifica parcialmente el Reglamento de Seguridad Privada, aprobado por Real Decreto 2364/ 1994, de 9 de diciembre.

Sobre libertad de radiodifusión se aprobó el Real Decreto $745 / 2001$, de 29 de junio, de adecuación del Real Decreto 991/1998, de 22 de mayo, por el que se crea el Consejo para las emisiones y retransmisiones deportivas (BOE n. ${ }^{\circ} 158$, de 3 de julio):

En materia de tutela judicial, el 7 de enero entró en vigor la Ley 1/2000, de 7 de enero (BOE n. ${ }^{\circ} 7$, de 8 de enero), de Enjuiciamiento Civil; por Real Decreto 1417/2001, de 17 de diciembre (BOE n. ${ }^{\circ} 310$, de 27 de diciembre), se procede a la conversión a euros de las cuantías establecidas en la Ley de Enjuiciamiento Civil. A través del Real Decreto $1162 / 2001$, de 26 de octubre (BOE ${ }^{\circ}{ }^{\circ} 258$, de 27 de octubre), se modifica el artículo 29 del Reglamento de asistencia jurídica gratuita.

Sobre el derecho a la educación, se aprobaron la Ley Orgánica 6/2001, de 21 de diciembre, de Universidades (BOE n. ${ }^{\circ} 307$, de 24 de 
diciembre); la Ley 5/2001, de 8 de mayo, sobre concesión de un suplemento de crédito, por importe de 4.866 .309 .925 pesetas, para el pago de retribuciones establecidas en diversas sentencias firmes, falladas a favor de las personas encargadas de la enseñanza de la religión católica en los colegios públicos (BOE $n .^{\circ} 111$, de 9 de mayo), y la Ley 13/2001, de 9 de julio, sobre concesión de un crédito extraordinario, por importe de 8.229.849.001 pesetas, para atender el pago de obligaciones derivadas de la compensación a las Universidades públicas por la reducción o exención en los precios públicos por servicios académicos a alumnos de familias numerosas correspondientes a los cursos 1996-1997 y 1997-1998 (BOE n. ${ }^{\circ} 164$, de 10 de julio).

Sobre derecho de petición se aprobó la Ley Orgánica 4/2001, de 12 de noviembre, reguladora del Derecho de Petición (BOE $n .^{\circ}, 272$, de 13 de noviembre).

En cuanto al servicio militar y la prestación social sustitutoria, por Real Decreto 247/2001, de 9 de marzo, se adelanta la suspensión de la prestación del servicio militar (BOE $n .^{\circ} 60$, de 10 de marzo); por Real Decreto 342/2001, de 4 de abril, se suspende la prestación social sustitutoria del servicio militar (BOE n. ${ }^{\circ} 60$, de 10 de marzo).

En materia de empleo y trabajo, se aprobaron el Real Decretoley 5/2001, de 2 de marzo, de medidas urgentes de reforma del Mercado de Trabajo para el incremento del empleo y la mejora de su calidad (BOE n..$^{\circ}$ 54, de 3 de marzo de 2001; corrección de errores: BOE n. ${ }^{\circ} 60$, de 10 de marzo; convalidación: 15 de marzo de 2001, BOE n. ${ }^{\circ}$ 72, de 24 de marzo de 2001), y la Ley 12/2001, de 9 de julio, de medidas urgentes de reforma del mercado de trabajo para el incremento del empleo y su calidad (BOE $n .^{\circ} 164$, de 10 de julio).

El Real Decreto 534/2001, de 18 de mayo, regula el acceso al empleo público de la Administración General del Estado y sus Organismos Públicos de nacionales de otros Estados a los que es de aplicación el derecho a la libre circulación de trabajadores (BOE $n .^{\circ} 130$, de 31 de mayo).

El Real Decreto 1466/2001, de 27 de diciembre (BOE $n .^{\circ} 311$, de 28 de diciembre), fija en 442,20 euros al mes el salario mínimo interprofesional para 2002.

En materia de Seguridad Social y jubilación, se aprobó el Real Decreto-ley 16/2001, de 27 de diciembre, de medidas para el establecimiento de un sistema de jubilación gradual y flexible (BOE $n .^{\circ} 313$, de 31 de diciembre; convalidación: 5 de febrero de 2002, BOE.$^{\circ} 38$, 
de 13 de febrero de 2002); por Real Decreto 1251/2001, de 16 de noviembre, se regulan las prestaciones económicas del sistema de la Seguridad Social por maternidad y riesgo durante el embarazo (BOE n. ${ }^{\circ}$ 276, de 17 de noviembre); el Real Decreto 1414/2001, de 14 de diciembre (BOE $n .{ }^{\circ} 311$, de 28 de diciembre), modifica el Real Decreto $728 / 1993$, de 14 de mayo, por el que se establecen pensiones asistenciales por ancianidad en favor de los emigrantes españoles; el Real Decreto 1464/2001, de 27 de diciembre (BOE n. ${ }^{\circ} 313$, de 31 de diciembre), aprueba la revalorización de las pensiones del sistema de la Seguridad Social para el ejercicio 2002; el Real Decreto 1465/2001, de 27 de diciembre (BOE $n .^{\circ} 313$, de 31 de diciembre), hace una modificación parcial del régimen jurídico de las prestaciones de muerte y supervivencia.

En materia de Sanidad y defensa de los consumidores se aprobó la Ley 11/2001, de 5 de julio, por la que se crea la Agencia Española de Seguridad Alimentaria (BOE $n .^{\circ} 161$, de 6 de julio).

A propósito de las profesiones colegiadas, el Real Decreto 658/ 2001, de 22 de junio, aprueba el Estatuto General de la Abogacía Española (BOE n. ${ }^{\circ} 164$, de 10 de julio) y por Real Decreto 936/2001, de 3 de agosto, se regula el ejercicio permanente en Espańa de la profesión de abogado con título profesional obtenido en otro Estado miembro de la Unión Europea (BOE n. ${ }^{\circ} 186$, de 4 de agosto).

En cuanto a la libertad de empresa y la defensa de la competencia, se aprobaron el Real Decreto-ley 2/2001, de 2 de febrero, por el que se modifica la disposición transitoria sexta de la Ley $54 / 1997$, de 27 de noviembre, del Sector Eléctrico, y determinados artículos de la Ley 16/1989, de 17 de julio, de Defensa de la Competencia (BOE n. ${ }^{\circ} 30$, de 3 de febrero; convalidación: 22 de febrero, BOE n. ${ }^{\circ} 51$, de 28 de febrero), y la Ley 9/2001, de 4 de junio, por la que se modifica la disposición transitoria sexta de la Ley 54/1997, de 27 de noviembre, del Sector eléctrico; determinados artículos de la Ley 16/1989, de 17 de julio, de Defensa de la Competencia, y determinados artículos de la Ley 46/1988, de 17 de diciembre, sobre introducción del euro (BOE n. ${ }^{\circ} 134$, de 5 de junio). El Real Decreto 1443/2001, de 21 de diciembre (BOE n. ${ }^{\circ}$ 16, de 18 de enero de 2002), desarrolla la Ley 16/1989, de 17 de julio, de Defensa de la Competencia en lo referente al control de las concentraciones económicas.

Por Real Decreto 1412/2001, de 14 de diciembre (BOE n. ${ }^{\circ} 13$ de 15 de enero de 2002), se modifica el Real Decreto 1251/1999, de 16 de julio, sobre sociedades anónimas deportivas. 
En materia medioambiental, se aprobó la Ley 6/2001 de 8 de mayo, de modificación del Real Decreto Legislativo 1302/1986, de 28 de junio, de evaluación de impacto ambiental (BOE $n .^{\circ} 111$, de 9 de mayo).

En el ámbito de la cultura, la Ley 15/2001, de 9 de julio, de fomento y promoción de la cinematografía y el sector audiovisual (BOE $n$. ${ }^{\circ}$ 164 , de 10 de julio).

En el ámbito jurisprudencial destacan, por lo que a los pronunciamientos del Tribunal Constitucional se refiere, los siguientes:

En materia de igualdad y no discriminación, en la STC 13/2001, de 29 de enero de 2001, se consideró que no se produjo una discriminación racial encubierta en la concreta forma de ejercicio de la potestad policial de identificación realizada por funcionarios de policía a la Sra. Williams Lecraft, "en su calidad de integrante de un grupo racial concreto... A tales efectos conviene recordar que, aun advirtiendo de la prudencia con la que deben usarse las referencias de carácter étnico para evitar malentendidos, su utilización con carácter descriptivo, en sí misma considerada, no resulta por principio discriminatoria (STC 126/1986, FJ 1). [Además] se desprende que la actuación policial usó el criterio racial como meramente indicativo de una mayor probabilidad de que la interesada no fuera española. Ninguna de las circunstancias acaecidas en dicha intervención indica que el comportamiento del funcionario de la Policía Nacional actuante fuese guiado por un prejuicio racista o por una especial prevención contra los integrantes de un determinado grupo étnico, como se alega en la demanda... Finalmente, no aparece tampoco acreditado que los funcionarios policiales desplegasen su actuación de forma desconsiderada, ofensiva o gratuitamente obstaculizante de la libertad de circulación de la demandante de amparo, pues la intervención policial se prolongó únicamente lo imprescindible para lograr la identificación. Finalmente puede descartarse que los agentes de policía actuasen de un modo airado o llamativo que hiciese pasar a la Sra. Williams Lecraft y sus acompañantes a un primer plano que les resultase afrentoso o incómodo frente a la colectividad de ciudadanos que hubiese en la propia estación de ferrocarril, sino que la toma de los datos de identidad se produjo en las dependencias policiales..." (FJ 9).

En la STC 20/2001, de 29 de enero, el Tribunal debía determinar si el cese de la recurrente en su condición de funcionaria interina durante el período en el que se encontraba disfrutando de un permiso por maternidad ha conculcado el derecho a la igualdad proclamado 
en el art. 14 CE y, muy especialmente, el derecho a no ser discriminada por razón de sexo establecido en el citado precepto constitucional.

Para el Tribunal, es patente el lazo causal que el organismo público estableció entre la situación de la trabajadora y la extinción de su relación funcionarial, con lo que se está reconociendo expresamente que la finalización anticipada de la situaçión funcionarial tuvo su única causa en la maternidad de la actora y en su consiguiente carencia de idoneidad física para el desempeño de sus funciones. Por todo ello, el cese de una funcionaria en régimen de interinidad por razón de su embarazo o por una causa basada esencialmente en ese estado sólo puede afectar a las mujeres, haciéndolas de peor condición que a los varones $y$, por lo tanto, constituye una discriminación basada en el sexo, sin que, como afirmamos en nuestra STC 240/1999, de 20 de diciembre (FJ 6), sea necesario (ni posible) aportar término de comparación.

La protección específica de la maternidad de los períodos pre y postnatales persigue una clara finalidad social, esto es, la protección de la maternidad, a la que se añade la finalidad de promocionar el establecimiento de una relación de carácter familiar, absolutamente necesaria con el que acaba de nacer. La protección extiende, por ello, su campo de actuación, alcanzado tanto al neonato como a la familia en su conjunto. Por ello, como en su día recordó la STC 203/2000, de 24 de agosto (FJ 5), "no resulta admisible, desde la perspectiva del art. $14 \mathrm{CE}$, fundar la denegación de un derecho con transcendencia constitucional (arts. 9.2 y $39.1 \mathrm{CE}$ ) exclusivamente en el carácter temporal y en la necesaria y urgente prestación del servicio propia de la situación de interinidad". De lo que se desprende la procedencia de otorgar el amparo solicitado... (FJ 3 y 6 ).

En materia de libertad religiosa, la STC 46/2001, de 15 de febrero, al estimar el amparo solicitado, entre otros, por la Iglesia de Unificación, concluye que "la articulación de un Registro ordenado a dicha finalidad no habilita al Estado para realizar una actividad de control de la legitimidad de las creencias religiosas de las entidades o comunidades religiosas, o sobre las distintas modalidades de expresión de las mismas, sino tan sólo la de comprobar, emanando a tal efecto un acto de mera constatación que no de calificación, que la entidad solicitante no es alguna de las excluidas por el art. 3.2 LOLR, y que las actividades o conductas que se desarrollan para su práctica no atentan al derecho de los demás al ejercicio de sus libertades y derechos fundamentales, ni son contrarias a la seguridad, salud o moralidad públicas, 
como elementos en que se concreta el orden público protegido por la ley en una sociedad democrática, al que se refiere el art. 16.1 CE. En consecuencia, atendidos el contexto constitucional en que se inserta el Registro de Entidades Religiosas, y los efectos jurídicos que para las comunidades o grupos religiosos comporta la inscripción, hemos de concluir que, mediante dicha actividad de constatación, la Administración responsable de dicho instrumento no se mueve en un ámbito de discrecionalidad que le apodere con un cierto margen de apreciación para acordar o no la inscripción solicitada, sino que su actuación en este extremo no puede sino calificarse como reglada" (FJ 8).

Sobre el derecho a la integridad física y a la protección de la intimidad, la STC 119/2001, de 24 de mayo, precisó que "cuando la exposición continuada a unos niveles intensos de ruido ponga en grave peligro la salud de las personas, esta situación podrá implicar una vulneración del derecho a la integridad física y moral (art. $15 \mathrm{CE}$ ). En efecto, si bien es cierto que no todo supuesto de riesgo o daño para la salud implica una vulneración del art. $15 \mathrm{CE}$, sin embargo cuando los niveles de saturación acústica que deba soportar una persona, a consecuencia de una acción u omisión de los poderes públicos, rebasen el umbral a partir del cual se ponga en peligro grave e inmediato la salud, podrá quedar afectado el derecho garantizado en el art. 15 CE».

Respecto a los derechos del art. $18 \mathrm{CE}$, debemos poner de manifiesto que en tanto el art. 8.1 CEDH reconoce el derecho de toda persona "al respeto de su vida privada y familiar, de su domicilio y de su correspondencia", el art. 18 CE dota de entidad propia y diferenciada a los derechos fundamentales a la intimidad personal y familiar (art. 18.1) y a la inviolabilidad del domicilio (art. 18.2). Respecto del primero de estos derechos fundamentales ya hemos advertido en el anterior fundamento jurídico que este Tribunal ha precisado que su objeto hace referencia a un ámbito de la vida de las personas excluido tanto del conocimiento ajeno como de las intromisiones de terceros, y que la delimitación de este ámbito ha de hacerse en función del libre desarrollo de la personalidad: De acuerdo con este criterio, hemos de convenir en que uno de dichos ámbitos es el domiciliario por ser aquél en el que los individuos, libres de toda sujeción a los usos y convenciones sociales, ejercen su libertad más íntima (SSTC 22/1984, de 17 de febrero, FJ 5; 137/1985, de 17 de octubre, FJ 2, y 94/1999, de 31 de mayo, FJ 5). Teniendo esto presente, podemos concluir que una exposición prolongada a unos determinados niveles de 
ruido, que puedan objetivamente calificarse como evitables e insoportables, ha de merecer la protección dispensada al derecho fundamental a la intimidad personal y familiar, en el ámbito domiciliario, en la medida en que impidan o dificulten gravemente el libre desarrollo de la personalidad, siempre y cuando la lesión o menoscabo provenga de actos u omisiones de entes públicos a los que sea imputable la lesión producida" (FJ 6).

En cuanto a la tutela judicial, la STC 115/2001, de 10 de mayo, plantea ante el Pleno del Tribunal cuestión de inconstitucionalidad respecto de los artículos 108, párrafo 2, de la Ley Orgánica 4/1987, de la Competencia y Organización de la Jurisdicción Militar, y 127, párrafo 1, de la Ley Orgánica 2/1989, de 13 de abril, Procesal Militar, por su contradicción con los arts. 14 y $24.1 \mathrm{CE}$, en relación con el inciso final del art. 117.5 de la Constitución.

A propósito del derecho al juez imparcial, la STC 69/2001, de 17 de marzo, aclara que, "en la medida en que la instrucción criminal, pese a su finalidad inquisitiva, obliga a consignar todas las circunstancias que puedan influir en la calificación de los hechos investigados, sean favorables o adversas al imputado, faculta para adoptar medidas cautelares que pueden afectar a derechos fundamentales de la persona $y$ debe respetar algunos principios (derecho de defensa, a conocer la imputación, de contradicción e igualdad entre las partes), se hace obligado que el instructor deba revestir las necesarias condiciones de neutralidad tanto en relación con las partes del proceso como sobre su objeto. En definitiva, el Juez de Instrucción, como cualquier Juez, debe ser un tercero ajeno a los intereses en litigio, a sus titulares y a las funciones que desempeñan en el proceso. En esta misma línea, cuando el Tribunal Europeo de Derechos Humanos ha analizado el contenido del art. 5.3 $\mathrm{CEDH}$, ha puesto de relieve, en una interpretación literal del precepto, que el ejercicio de funciones judiciales no se limita necesariamente a juzgar, $y$, de otro lado, desde una interpretación sistemática del Convenio, ha observado que la finalidad de la norma es la de garantizar la imparcialidad y la objetividad de toda persona que ha de decidir sobre la privación de la libertad de otra. $Y$, en este sentido, el Juez o la "autoridad habilitada por la ley" debe de ser independiente del poder ejecutivo y de las partes, dada su obligación de examinar las circunstancias que actúan en favor o en contra del arresto. A ello cabe añadir que, aun cuando el mismo Tribunal ha declarado que nada impide que la autoridad competente para acordar la detención pueda desempeñar otras funciones (a título de ejemplo, su intervención procesal posterior como parte acusadora), la actuación 
de dicha autoridad correrá el riesgo de inspirar a los justiciables dudas legítimas sobre su imparcialidad si no es revisada por el juez encargado del caso (en este sentido se pronuncian las SSTEDH de 4 de diciembre de 1979, caso Schiesser; de 22 de mayo de 1984, casos Jong y otros, Van der Sluijs y otros y Duihof y Duijf; de 26 de mayo de 1988, caso Pauwels; 30 de marzo de 1990, caso Huber; de 26 de noviembre de 1992, caso Brincat; de 28 de octubre de 1998, caso Assenov, y de 24 de julio de 2000, caso Niebdala).

Así pues, aunque el contenido de la garantía constitucional de imparcialidad del Juez de Instrucción, dada la configuración de nuestro sistema procesal, no sea idéntica a la que pueda predicarse del órgano de enjuiciamiento (pues habrá de ponerse en conexión con las resoluciones o determinaciones que concretamente haya adoptado en un determinado asunto), es también exigible a aquél en la medida en que en esta fase del proceso penal, tal y como viene diseñado en nuestras leyes procesales, ha de resolver las pretensiones que ante él se formulen sin prejuicios ni motivaciones ajenas a la recta aplicación del Derecho, $y$ ha de tomar determinaciones que pueden afectar a los intereses o derechos fundamentales de las partes (así ocurre con los Autos de prisión o libertad provisional, de procesamiento, de sobreseimiento o de apertura del juicio oral en el procedimiento abreviado, por ejemplo), sobre las cuales ha de exigirse la previa condición de que el . Juez que las adopte aparezca tanto subjetiva como objetivamente neutral» (FJ 17). 